\title{
CORRECTION
}

\section{Correction to: Are introduced plants a threat to native pollinator services in montane-alpine environments?}

\author{
Christa M. Miller ${ }^{1} \cdot$ Barbara I. P. Barratt ${ }^{1,2} \cdot$ Katharine J. M. Dickinson $^{1} \cdot$ Janice M. Lord ${ }^{1}$
}

Published online: 28 June 2018

(c) Swiss Botanical Society 2018

\section{Correction to: Alpine Botany https://doi.org/10.1007/s00035-018-0206-5}

In the original publication, latitude and longitude values are incorrect in Table 1. The corrected Table 1 is given below.

Table 1 Study site characteristics, the Remarkables range, south-central South Island, New Zealand

\begin{tabular}{|c|c|c|c|c|c|c|c|}
\hline \multirow[t]{2}{*}{ Elevation m.a.s.1 } & \multirow[t]{2}{*}{ Latitude } & \multirow[t]{2}{*}{ Longitude } & \multirow[t]{2}{*}{ Season } & \multicolumn{2}{|c|}{ Flowering species } & \multicolumn{2}{|c|}{ Network parameters } \\
\hline & & & & Native & Introduced & Network size & $\begin{array}{l}\text { Qualitative- } \\
\text { connectance }\end{array}$ \\
\hline \multirow[t]{2}{*}{647} & \multirow[t]{2}{*}{$-45.025580^{\circ}$} & \multirow[t]{2}{*}{$168.768074^{\circ}$} & 2011-2012 & 1 & 3 & 40 & 22.5 \\
\hline & & & 2012-2013 & 1 & 4 & 50 & 34.0 \\
\hline \multirow[t]{2}{*}{903} & \multirow[t]{2}{*}{$-45.025690^{\circ}$} & \multirow[t]{2}{*}{$168.777629^{\circ}$} & 2011-2012 & 1 & 2 & 21 & 52.4 \\
\hline & & & 2012-2013 & 3 & 3 & 60 & 26.7 \\
\hline \multirow[t]{2}{*}{1114} & \multirow[t]{2}{*}{$-45.026881^{\circ}$} & \multirow[t]{2}{*}{$168.803354^{\circ}$} & 2011-2012 & 5 & 9 & 140 & 23.6 \\
\hline & & & 2012-2013 & 7 & 7 & 112 & 24.1 \\
\hline \multirow[t]{2}{*}{1315} & \multirow[t]{2}{*}{$-45.033388^{\circ}$} & \multirow[t]{2}{*}{$168.801054^{\circ}$} & 2011-2012 & 6 & 4 & 130 & 27.7 \\
\hline & & & 2012-2013 & 8 & 3 & 110 & 28.2 \\
\hline \multirow[t]{2}{*}{1464} & \multirow[t]{2}{*}{$-45.043479^{\circ}$} & \multirow[t]{2}{*}{$168.804554^{\circ}$} & 2011-2012 & 9 & 3 & 120 & 25.8 \\
\hline & & & 2012-2013 & 6 & 4 & 150 & 34.0 \\
\hline
\end{tabular}

Flowering species are the number of species used for flower visitor observations within each study plot in each season. "Network size" is the number of flowering species $\times$ number of insect taxa observed visiting flowers. "Qualitative connectance" is $100 \times$ (the number of unique plantinsect interactions/network size). Network parameters are derived from pooled weekly observations from 20 weeks in the 2011-2012 flowering season and 24 weeks in the 2012-2013 flowering season. As not all insect taxa were identified beyond the family level, these are minimum network parameters

The original article can be found online at https://doi.org/10.1007/ s00035-018-0206-5.

Janice M. Lord

janice.lord@otago.ac.nz

1 Department of Botany, University of Otago, Dunedin, New Zealand

2 AgResearch, Invermay Agricultural Centre, Mosgiel, New Zealand 